\section{Binding of Estrone to Microfiltration Hollow Fibre Membranes in Filtration of Solutions Containing Trace Estrone}

*Sheng Chang, * T. David Waite, *Andrea I. Schäfer, **Anthony G. Fane, *Centre for Water and Waste Technology, School of Civil and Environmental Engineering, UNSW, Sydney, NSW 2052, Australia, D.Waite@unsw.edu.au *UNESCO Center for Membrane Science and Technology, School of Chemical

Engineering and Industrial Chemistry, UNSW, Sydney, NSW 2052, Australia,

\section{ABSTRACT}

Increased concern is being paid to the health and environmental risk caused by trace natural and synthetic hormones discharged from sewage treatment plant (STPs). This study, which is part of a larger project on investigation of hybrid membrane processes for study, which is part of a larger project on investigation of hybrid membrane processes for
trace hormones removal, focuses on binding of hormones to microfiltration hollow fibre membranes in filtration of solutions containing trace hormones. The adsorption capacity of the membrane, kinetics of adsorption and desorption of to/from microfiltration hollow fibre membranes, and the factors affecting adsorption to the membranes have been studied using estrone as model solute. The experiments showed that the adsorption of estrone to the microfiltration membranes could result in high retention of estrone molecules. sieving, the retention decreases with increase in the amount of estrone accumulated on the membrane and breakthrough occurs when the accumulated estrone on the membrane reaches an equilibrium concentration corresponding to the feed concentration. For long-term operation, although membranes could be saturated by hormone molecules the membrane processes could still have a function of buffer for instantaneous high hormones concentration because the saturation surface concentration increased with the increase in the feed concentration. In addition, the experiments also indicated that the adsorption capacity of the membranes for hormones could be affected by membrane types, $\mathrm{pH}$, affinity of hormones to water, as well as the presence of other organics. These results are affinity of hormones to ware of relevance to the potential release of trace hormones from water treatment systems

\section{KEY WORDS:}

Microfiltration; trace organics; hybrid membrane process; adsorption, hormones,

\section{INTRODUCTION}

Although most of natural and synthetic hormones could be removed through biodegradation or sorption in the STP processes the remaining trace compounds in the effluent, usually in the range of $1-30 \mathrm{ng} / \mathrm{L}$, still have significant effect on biota exposed in the receiving the receiving envire consequences caused by these compounds include feminisation of male fish [Sumpter and Jobling, 1995], increased risk of testicular cancer and decrease in sperm quantity and quality in human [Sharpe and Skakkebaek, 1993].
Further removal of such a low concentration of organic compounds in an economical way is an engineering challenge. One of the possible solutions to this problem is to combine low-pressure membrane processes such as UF or MF with other physicochemical processes in so-called hybrid membrane processes. Hybrid membrane processes involving physicochemical steps, such as activated carbon adsorption and coagulation, have been shown to be highly effective in removal of dissolved constituents in some instances [Best et al, 1999; Kim et al, 1996].

This study, which is part of a larger project on investigation of hybrid membrane processes for trace hormones removal, was stimulated by preliminary finding that nearly $100 \%$ estrone was lost on passage of solutions containing low concentrations of this hormone through hydrophobic microfiltration hollow fibre membranes. In our previous studies, the adsorption capacity of the hydrophobic membrane for estrone and the adsorption rate have been assessed using batch adsorption experiments which were carried out by placing a selected amount of hollow fibre membranes into a number of flasks which contained estrone solution of a specific initial concentration. The experimental models for adsorption capacity and kinetics have been developed in the studies. In the present paper, we focused on the binding of estrone to the microfiltration hollow fibres in filtration of solutions containing trace estrone. Membrane retention of estrone caused by dynamic adsorption and retention breakthrough due to membrane saturation are discussed. In addition, the factors affecting estrone adsorption to the membranes, including $\mathrm{pH}$ membrane characteristics, and the presence of secondary organics are also discussed.

\section{EXPERIMENTAL MATERIALS AND METHODS}

Flask adsorption and dead-end filtration tests were carried out to assess the adsorption characteristics of estrone on hollow fibre membranes. The flask experiments were carried out in an incubator (Bioline, Edwards Instrument Company Australia) under conditions of $250 \mathrm{rpm}$ and $25^{\circ} \mathrm{C}$. For dead-end filtration, a small bundle of hollow fibre membranes was directly immersed in the test solutions and a peristaltic pump (Masterflex 7518-00, ColeParmer Instrument Company) was used to pump the permeate out from the fibre lumen Most of adsorption experiments were carried out using a hydrophobic $0.2 \mu \mathrm{m}$ USFILTER hollow fibre membrane but a hydrophilic Zenon $0.02 \mu \mathrm{m}$ hollow fibre membrane was also cxamined for comparison. The mean outer and 0.02 um hollow fibre member was a examined for membrane, respectively. All the hydrophobic membranes tested were wetted by $50 \%$ alcohol solution before experiments.

$1 \mathrm{~m} \mathrm{M} \mathrm{NaHCO}$ and $20 \mathrm{mM} \mathrm{NaCl}$, which provided buffering to about $\mathrm{pH} 8$, surface water and secondary effluent were used as the background solution. The surface water and secondary effluent have TOCs of 5.7 and $13.2 \mathrm{ppm}$, respectively. The test solutions were prepared by dissolving a certain amount of ${ }^{3} \mathrm{H}$-labelled estrone in the background prepared by dissol solutions. The radiolabeled estrone was purchased from Sigma Aldrich (Saint Louis,
Missour, USA). A Packard Instruments scintillation counter which has a detection limit of Missour, USA). A Packard Instruments scintillation counter which has a detecti
approximately $0.1 \mathrm{ng} / \mathrm{L}$ was used for analysis of the radiolabeled compounds.

\section{RESULTS AND DISCUSSION}

Batch Adsorption

In our previous study, the adsorption capacity of the hydrophobic membrane for estrone was assessed by batch adsorption experiments [Chang et al]. The batch adsorptions were carried out by placing a selected amount of hollow fibre membranes into a number of 
flasks which contained estrone solution of a specific initial concentration and shaking the mixture for 24 hours in the incubator at constant temperature. The experiments showed the partitioning of estrone at the equilibrium concentrations for the tested buffer solution can be correlated by Freundlich equation in the concentration range of $1.3 \mathrm{ng} / \mathrm{L}$ to $42 \mathrm{ng} / \mathrm{L}$ (Figure 1).

$$
C_{s e}=3.39 C_{e}^{1.30}
$$

Estimation of the surface area provided by the hollow fibre membranes shows that nearly all of the surface area is provided by the internal pore surface, so adsorption presumably occurs mainly on the internal pore surface. The transport steps involved in adsorption of estrone to the membrane surfaces include: transport in the bulk solution, boundary layer film transport, internal pore diffusion, and adsorption. Although it is usually assumed that the rate of adsorption is controlled by one or more of these transport processes, the batch adsorption experiments also exhibited that there is an obvious relationship between the adsorption rate and the degree of saturation of the membrane by estrone. Based on an assumption that the rate of adsorption is proportional to the site availability, which is defined as the difference between the surface concentration at the equilibrium state and that as time $\mathrm{t}$, to a power of $\mathrm{n}$, we developed an experimental kinetic model for batch adsorption of estrone to the hydrophobic hollow fibre membranes [Chang et al].

$$
C_{s t}=C_{s e}\left(1-\frac{1}{\left(0.028 C_{s e}^{0.05} t+1\right)^{1.818}}\right)
$$

Figure 2 shows that the kinetic behavior for adsorption of estrone to the membranes with different initial concentrations could be described by this developed model very well.

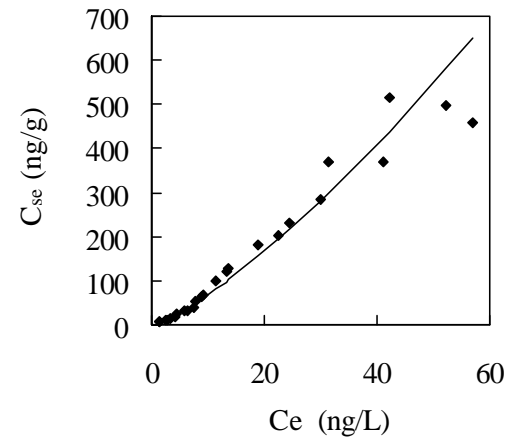

- Experimental — Model

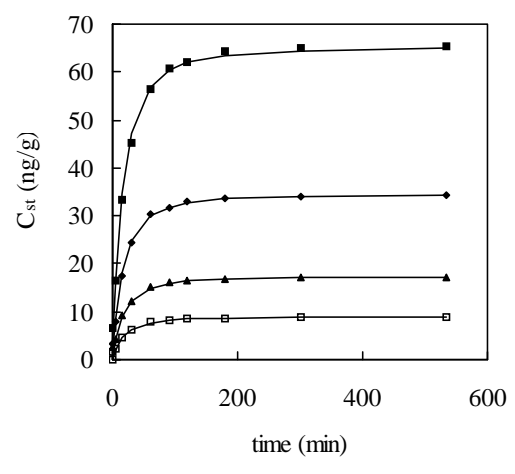

- $8 \mathrm{ng} / \mathrm{L} \quad \triangle 15 \mathrm{ng} / \mathrm{L} \quad$ • $30 \mathrm{ng} / \mathrm{L}$
Figure 1 Experimental and predicted isotherm relationships.

Figure 2 Experimental and simulated kinetic behavior.

In the present study, batch adsorption experiments with a certain flux were carried out to assess the effect of the permeate flow on adsorption capacity and kinetics. In the batch adsorption with flux studies, the permeate was pumped out of the fibre lumen and then returned to the feed flasks. The feed flask was slightly shaken each time before taking a sample from it to ensure that the sample reflected the average concentration of the solution contained within. Figure 3 shows the change in estrone concentration of the solutions with time and the final equilibrium concentration achieved after 24 hours adsorption for adsorptions with and without flux. From this figure it can be seen that the rate of adsorption with the permeate flow rate of $0.45 \mathrm{~g} / \mathrm{min}$ was slightly lower than those with the higher permeate flow rate and flask shaking. Since permeate flow is expected to favor estrone transport inside the pore but is not as effective as shaking in assisting bulk transport the lower attributed to bulk transport limitation. Given that nearly all of the adsorption capacity was provided by the internal pore surface, the higher adsorption rate with flask shaking suggested that the estrone molecules could be effectively transported to the adsorption site through boundary layer film transport and internal pore diffusion if they can be delivered to the mouths of the membrane pores. On the other hand, although a difference in adsorption kinetics for adsorption with and without flux was observed, the comparison of the final solution concentration after 24 hours showed that the flux had negligible effect on the adsorption capacity of the membrane for estrone.

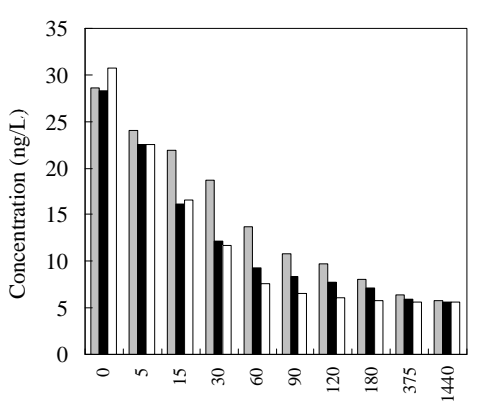

time (min)

$\square 0.45 \mathrm{~g} / \mathrm{min}$

$1.575 \mathrm{~g} / \mathrm{min} \quad \square$ shaking

Figure 3 Batch adsorption of estrone with and without flux.

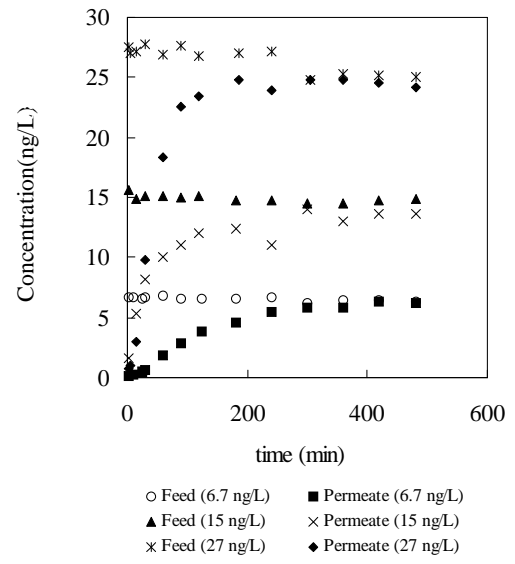

Figure 4 Change in estrone concentration in permeate and feed sides

\section{Filtration of solutions containing trace estrone}

The continuous dead-end filtrations of estrone solutions were carried out by immersing a small bunch of hollow fibres into a feed tank which had a volume of $800 \mathrm{ml}$ and the permeate was continuously pumped out of the fibre lumen at a certain flow rate. Figure 4 shows that the change in estrone concentration with time in the feed and permeate during continuous dead-end filtration of estrone solutions of different concentrations. It can be seen that the estrone concentration in the permeate increased with time and gradually became close to the concentration in the feed, suggesting that the membrane rejection decreased with the accumulation of estrone on the membranes and tended to reach breakthrough.

For the continuous dead-end filtration of solutions containing trace estrone, it can be assumed that an instantaneous adsorption equilibrium is attained on the passage of the solutions through the membranes. Based on such an assumption, the rejection of the membrane depends on the equilibrium partitioning of estrone between the membrane and 
the solutions. The higher the surface concentration, the higher the permeate concentration. When the amount of estrone accumulated on the membrane surface reaches the equilibrium surface concentration corresponding to the feed estrone concentration the membrane could be regarded to be saturated by estrone molecules and the concentration in the permeate side will equal that in the feed side. The time taken to saturate the membrane can be estimated by the developed experimental model (Eq. 2). Since $\mathrm{Eq} 2$ is only valid for $\mathrm{C}_{1} \neq \mathrm{C}_{\mathrm{e}}$ we assume the membrane will become saturated when $C_{t}=95 \%$ of $C_{e}$. Then: $t_{e}=150 C_{s e}^{-0.05}$

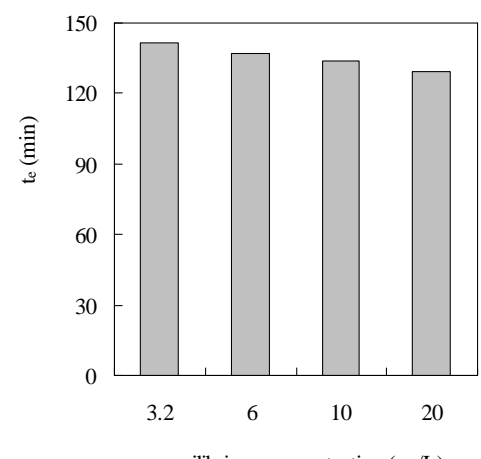

equilibrium concentration $(\mathrm{ng} / \mathrm{L})$

\section{Figure 5 Simulated time taken for membrane} saturation
(3)

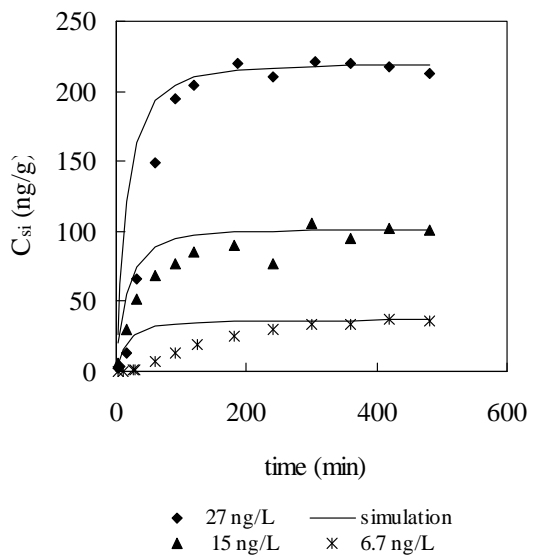

Figure 6 The accumulation of estrone on the membrane surface
Figure 5 shows the simulated time taken for saturation of the membrane under different equilibrium concentrations. From this figure it can be noted that although $t_{e}$ decreased with the increase in $\mathrm{C}_{\mathrm{se}}$ the final equilibrium concentrations, in fact, have negligible effect on $\mathrm{t}_{\mathrm{e}}$ over the normal concentration range. However, it is worth noting that equation 3 was developed from the experimental kinetic model for batch adsorption (Eq.2) where bulk transport was not a limiting step due to vigorous shaking. For dead-end filtration, the bulk transport could limit the rate of adsorption and the membrane may take longer to become saturated. Figure 6 compares the kinetic behavior of the adsorption of the continuous dead-end filtration for different feed concentrations with those predicted by the experimental model for batch adsorption. From the figure it can be seen that the adsorplon ates for deadend $6.7 \mathrm{nglt}$ solution, ind model, particularly for the $6.7 \mathrm{ng} / \mathrm{L}$ solution, indicating that adsorption for the dead-end filtration case is dominated by molecular diffusion in bulk solution. However, the time calculated by Equation 3 could be regarded as the minimum time required to render the membrane saturated.

Although for long-term operation the membrane could be saturated by hormone and provide essentially no rejection to these organic molecules, the membrane could still provide an effective buffer to the instantaneous fluctuation of hormone concentration due to the increased adsorption capacity of the membrane to hormone on increase in hormone concentration in the influent. Figure 7 shows the concentrations of the permeate and feed on successive filtration of estrone solutions of different concentrations using the same membrane bunch during the filtration runs. In these experiments, estrone solutions which had concentrations of $668,155,68,35,14,566,159 \mathrm{ng} / \mathrm{L}$, were successively passed through the $0.2 \mu \mathrm{m}$ membranes. For each solution the filtration lasted for 30 minutes and the samples for the retention assessment for each solution were taken at 15 minutes after the filtration commenced. Form this figure it can be seen that, although the concentration of the estrone in the feed side change over a wide range, the concentration in the permeate side was maintained relatively stable. This is because the adsorption capacity of the membrane increased with the increase in feed concentration. However the results shown in figure 7 also indicate that the adsorption of estrone to the membrane surface is reversible. This suggests that the accumulated additional estrone on the membrane surface caused by instantaneous high concentration will be gradually desorbed from the

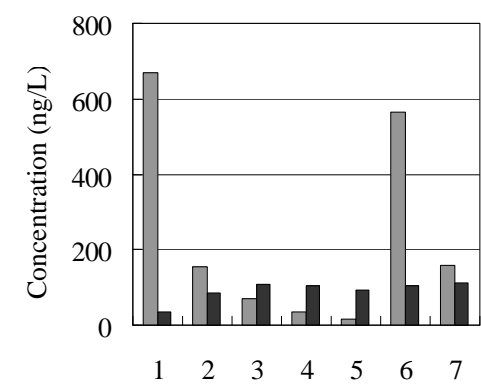

Order of filtration

$$
\square \text { feed } \square \text { permeate }
$$

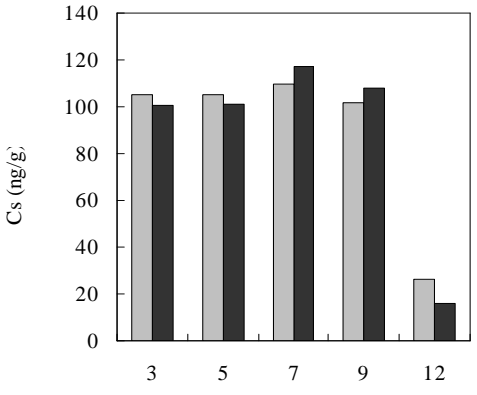

$\mathrm{pH}$

\section{Figure 7 Successive filtration of estrone solutions Figure $8 \mathrm{Effect}$ of $\mathrm{pH}$ and ionic strength}

\section{Factors Affecting Estrone Adsorption to Membrane}

Effects of $\mathrm{pH}$ and ionic strength: Figure 8 shows the removal of the estrone by the same amount of hollow fibre membranes in two hour batch adsorption runs under different $\mathrm{pH}$ and ion strength conditions. From this figure, it can be seen that there is no significant difference in the estrone removal over a pH range of 3 to 9 for sodium chloride concentrations of 0.02 to $0.2 \mathrm{M}$. However, for $\mathrm{pH} 12$ an obvious decrease in extent of removal of estrone was observed. Figure 9 compares the adsorption capacity of the hollow fibre membranes under different $\mathrm{pH}$ conditions with those predicted by Equation 1. This also shows that the adsorption capacity at $\mathrm{pH} 12$ is much lower than the values predicted by the experimental model. The tendency of the estrone to be adsorbed is a function of its affinity for water as compared to its affinity for the membrane. When the $\mathrm{pH}$ is higher than 11 , the estrone is expected to become charged as a result of deprotonation resulting in an increase in hydrophilicity and a lowered affinity for the membrane.

Effect of membranes: Figure 10 compares the adsorption capacity of the Zenon membrane (hydrophilic) and USfilter membrane (hydrophobic) for estrone based on unit 
membrane surface area. From this figure it can be seen that the adsorption capacity of the USFilter membrane is much higher than that of the Zenon membrane. One of the possible reasons for such a difference is the relative hydrophobicity of the membranes. It can be expected that the more hydrophilic membrane will possess a higher affinity for water molecules with less binding sites available for the estrone molecules. This may reduce the adsorption capacity of the hydrophilic membrane to the estrone.

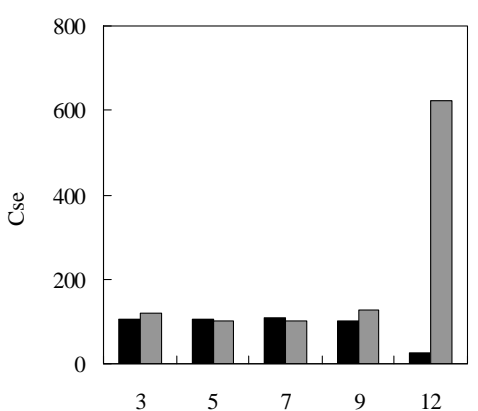

$\mathrm{pH}$

$0.02 \mathrm{M} \quad \square$ Model

Figure 9 Experimental and simulated adsorption capacity under different $\mathrm{pH}$ conditions.

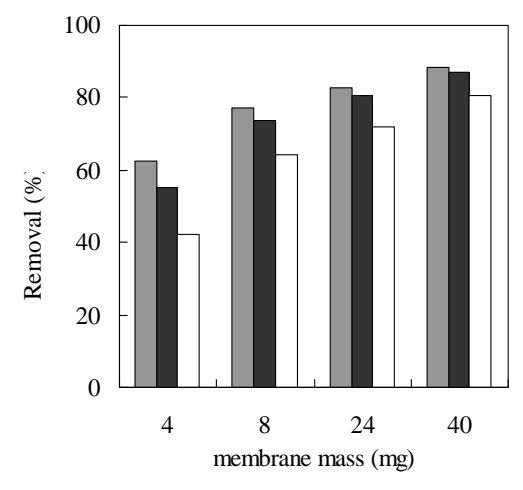

Figure 11 Effect of background solution. (buffer: $\mathrm{pH} 8$, surface water: $\mathrm{pH} 7.0$, Secondary effluent: $\mathrm{pH} 7.2-7.5$ )

$\square$ buffer $\square$ surface water $\square$ secondary effluent

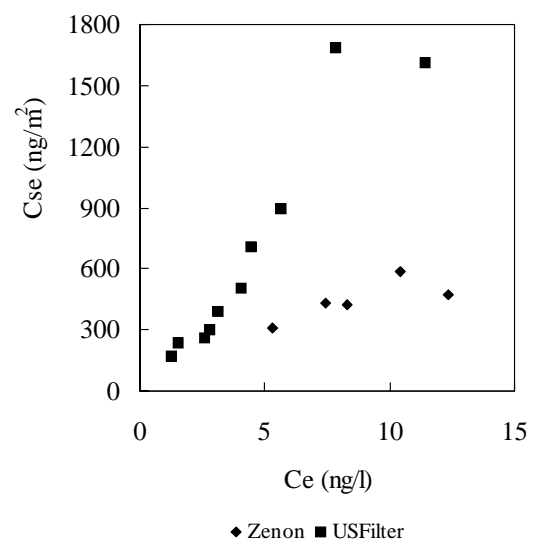

Figure 10 Adsorption capacity of USfilter and Zenon membranes to estrone assesed by testing membrane adsorption capacity for estrone in different backgrous assutions: carbonate buffer solutions: carbonate buffer, natural surface water (TOCs $5.7 \mathrm{ppm}$ ) and secondary effluent (TOCs $13.2 \mathrm{ppm}$ ). Figure 11 shows the removal of estrone by different amount of hollow fibre membranes from different background solutions. From this figure it can be seen that the removal of estrone from surface water and the secondary effluent is slightly lower than from the buffer solution, suggesting that the adsorption capacity of the membrane to the estrone may be reduced by competitive adsorption of other hydrophobic organics. The effect however is not large and indicates that the presence of other solution species does not dramatically influence estrone partitioning to the membrane.

\section{CONCLUSIONS}

This study provides extensive insight into the binding of hormones to microfiltration hollow fibre membranes in microfiltration of solutions containing trace hormones. The partitioning of estrone between membrane and solution at the equilibrium state, membrane retention to estrone and retention breakthrough, and factors affecting adsorption capacity of the membrane have been discussed. The experiments showed that adsorption could result in a significant accumulation of estrone on the hydrophobic membrane surface and a high retention of estrone by microfiltration membranes. The retention of the membrane decreases with the increase in the surface concentration of estrone and a breakthrough will occur when the surface concentration reaches the equilibrium value corresponding to the feed concentration. On the other hand, the assessment of factors of membrane to estrone showed that the adsorption capacity of estrone could be reduced by high $\mathrm{pH}$, increase in affinity of both of membranes and hormone molecules for water, as well as presence of other hydrophobic organics. These results provide insight into the potential release of trace hormones from water treatment systems where microfiltration membranes are used as a process barrier.

\section{ACKNOWLEDGMENT}

Funding for this research was provided by Queensland Government, Australia and the Australian Research Council.

\section{REFERENCES}

Best, G., Mourato, D., Singh, M., 1999, Application of immersed ultrafiltration membranes for color \& TOC removal. Membrane Technology 112, 5 -12.

Chang, S., Waite, T. D., Schäfer, A. I., Fane, A. G., Adsorption of trace hormones by hydrophobic microfiltration hollow fibre membranes. Water Research (in preparation).

Johnsin, A. C., Sumpter, J. P., Removal of endocrine-disrupting chemicals in activated sludge treatment works, Environmental Science and Technology (in press).

Kim, J. S., Lee, S. J., Yoon, S. H., and Lee, C. H., 1996, Competitive adsorption of trace organics on membranes and powdered activated carbon in powdered activated carbon ultrafiltration system. Wat. Sci. Tech.,34 (9), $223-229$

Sharpe, R. M., Skakkebabaek, N. E., 1993, Are estrogens involved in falling sperm counts and disorders of the male reproductive tract? Lancet 341, 1392 - 1395

Sumpter, J. P., Jobling, S., 1995, Vitellogenesis as a biomarker for estrogenic contamination of the aquatic environment. Environ. Health Perspect 103, $173-178$.

\section{CONTACT}

Professor T. D. Waite

Centre for Water and Waste Technology,

School of Civil and Environmental Engineering,

UNSW, Sydney, NSW 2052, Australia,

D.Waite@unsw.edu.au, Tel: 61-2-93855060, Fax: 61-2-93856139 